Original scientific paper

\title{
FUSED DEPOSITION MODELLING FOR 3D PRINTING OF SOFT ANTHROPOMORPHIC ACTUATORS
}

\author{
Curkovic, P.* \& Cubric, G. ${ }^{* *}$ \\ * University of Zagreb, Faculty of Mechanical Engineering and Naval Architecture, Ivana Lucica 5, \\ 10000 Zagreb, Croatia \\ ${ }^{* *}$ University of Zagreb, Faculty of Textile Technology, Prilaz baruna Filipovica 28, 10000 Zagreb, \\ Croatia \\ E-Mail: petar.curkovic@fsb.hr, goran.cubric@ttf.hr
}

\begin{abstract}
Soft actuators are increasingly gaining attention in the robotics community due to several favourable properties rooted mainly in their inherent compliance. They are safe, can easily grasp different objects, and cheap to manufacture. However, particularly in the case of FDM (Fused Deposition Modelling) printed actuators, their design is usually an iterative process that relies on intuition due to complex material models, nonlinearities, large deformations, and residual stresses caused by imperfect interlayer structure. This makes such actuators difficult to model and control and limits their implementation despite some comparative advantages over the other 3D printing technologies. In this study, to characterize the properties of FDM printed soft actuators, we compare a simple but computationally effective linear model with a realistic experimentally generated hyperelastic material model of a soft actuator. Based on these insights, we 3D print a fully operational soft anthropomorphic hand and use it in a set of experiments to evaluate the limitations of the models and suggest design and printing parameters to improve soft actuators' performance.

(Received in February 2021, accepted in April 2021. This paper was with the authors 2 weeks for 2 revisions.)
\end{abstract}

Key Words: Soft Robots, Modelling, Analysis, Fused Deposition Modelling, Anthropomorphic Actuator

\section{INTRODUCTION}

Soft robotics is an increasingly growing filed that focuses on design and manufacture of robots out of soft materials. The field is combining robotics, chemistry, and mechanics of materials to enable the preprograming of function - complex motion into flexible, soft materials $[1,2]$. The term soft refers to materials with Young's modulus $\sim 10^{4}-10^{9} \mathrm{~Pa}$, which is comparable to biological tissues such as skin, muscles, and to a lesser extent bone. Traditional robots made of metal alloys have elastic moduli in the range $\sim 10^{9}-10^{12} \mathrm{~Pa}$. Engineering materials such as silicones, hydrogels, rubber, thermoplastics, fit well into the range of soft materials which makes them suitable for soft robotics applications [3]. The actuation of these soft structures can be achieved by various stimuli, including pressure of fluids, both pneumatics and hydraulics, electrical charges for electroactive polymers, chemical reactions, shape memory alloys, and magnetic effects $[4,5]$.

Applying materials with low and variable stiffness has a consequence of large structural strains during operation. In case of soft robots, this is favourable, since it enables different applications such as grasping [6,7], locomotion and sensing [8]. Unlike conventional robots which are functional in a highly defined environment, soft robots provide much more flexibility, they are resistant to impacts, uncertainties, and can operate in loosely defined environments [9], [10]. In the case of multi-material design of soft robots' body, increased flexibility in functional preprograming is achievable [11]. When it comes to grasping, soft robots conform to shape, eliminating occurrence of concentrated pressure that can lead to object damage. This makes them safe for grasping soft and sensitive objects as well as objects whose shape is unknown. 
The absence of rigid component makes soft robots inherently safe for interactions with humans. This opens many new paths for their application. In the medical robotics field, soft actuators are used for design of wearable devices that improve rehabilitation process for patients with diseases such as arthritis, cerebral palsy, Parkinson's disease, and stroke $[12,13]$. Continuum kinematics and ability to navigate through narrow spaces without damaging the surrounding environment makes them ideal candidates for minimally invasive surgery, particularly in the field of endoscopy [14].

Recent applications of soft robots indicate the trend of their merging with textiles to produce functional clothing - wearable robotic garments. These novel products are paving the way for providing i.e., locomotion assistance, thermoregulation, shape change for assisted dressing, and therapeutic compression [15-17].

A particularly important task in the mentioned applications is to characterize the relation of activation stimuli to the behaviour of the soft actuator. To do so, suitable methods are required, but currently scarcely available. Additionally, expert knowledge is required to make use of those available. This limits the wider penetration of soft robotic systems in real-life industrial applications. The filed is now expanding, and the problems included in modelling of such highly nonlinear properties are numerous, demanding, and not readily applicable to various structures, different materials, and various processes used to manufacture soft actuators.

Several recent studies exist which are focused on numerical modelling of soft actuators, including complex algorithms able to capture their hyperelastic nature, viscous phenomena, and other nonlinearities $[18,19]$. Although important, their implementation requires a prominent level of knowledge in continuum mechanics and a customization of Abaqus environment to be implemented. In addition, these approaches are related the actuators manufactured by casting, and not FDM manufactured components. There is a significant difference between the two, since casted parts do not exhibit pronounced porosity which proved to be a primary source of deviation between models and experiment in this study.

There are a significantly fewer number of studies involved in both modelling and application of FDM for soft robotics applications. Data for such materials are not provided, which could be directly used to create realistic material models of their highly non-linear behaviour. It is shown in this study, but also others, that there is a slight difference of data given by manufacturers, and those obtained experimentally on a universal testing machine of processed filament for printed specimens. This is limiting wider application of FDM manufactured soft actuators in real world applications [20].

A recent study [21] considers 3D printed NinjaFlex specimens (which is the filament used for soft parts of the hand presented in this study), with several constitutive models fitted to experimentally obtained data. Ogden model was finally adopted and used for simulation of soft actuators, and a good correlation was presented. Experiments performed in another study [22] where the focus was set on mechanical characterization of NinjaFlex, but without further soft robotics application also suggest the use of Ogden hyperelastic model. Although both studies suggest the same model, there is a difference in material model parameter values, even though same filament was used. This suggests that additional characterization of material is necessary. Also, data experimentally determined in both studies are not in a perfect agreement with those given by the manufacturer [23].

Several recent studies consider the effect of voids on fused deposition modelled parts $[24,25]$. The results are focused on experimental computational tomography testing and statistical modelling of voids depending on different printing parameters. Although significant, results are focused on static load cases, and are not directly applicable to more complex conditions including bending, and high curvatures which occur in soft actuators. Finite volume method in combination with finite element method for composite structure analysis including 
trapped air voids is proposed in [26]. This approach has potential application for analysis of thermally activated soft actuators in 4D printing domain which is now rapidly expanding.

In this study, we use Thermoplastic polyurethane (TPU) based filament, proprietary available under name NinjaFlex to create soft parts of the proposed anthropomorphic hand. To model the dependency of bending in respect of activating pressure, and create initial distribution of air chambers, a simple linear model is used. To enable more realistic modelling, we perform experimental tensile strength test of specimens, and use these data to formulate a set of hyperelastic material models in Simulia Abaqus. Finally, we 3D print fingers in soft material and palm manifold in rigid material and assemble a fully functional soft anthropomorphic hand. The hand is then used to do experimental comparison of the linear model, hyperelastic model, and the physical model of the hand. We show that the properties of soft parts of the hand, primarily porosity, which proved to be the most influential parameter in deviation of models and experiment, depend significantly on the parameters used for $3 \mathrm{D}$ printing. We indicate parameters which yielded best properties in terms of reduced porosity, increased surface quality, reduced stringing, and reduced rigidity.

\section{MATERIALS AND METHODS}

We propose a design of the anthropomorphic hand with a rigid-soft structure. In doing so, we can experimentally verify material models used for simulation of soft parts of the hand. The outline of design procedure is schematically presented in Fig. 1.

The model of a human hand was used as a rough guide for dimensional proportions. Geometrical transformations were applied to remove or separate the parts which will not be printed or will be printed in different materials. Fingers are to be printed in soft material, and the palm manifold in rigid material. The surfaces for bonding of fingers to the palm are designed with maximizing the contact area in mind to reduce air leaking. The internal structure is designed to allow all the fingers to be independently actuated by integrating five air channels into the manifold of the palm.

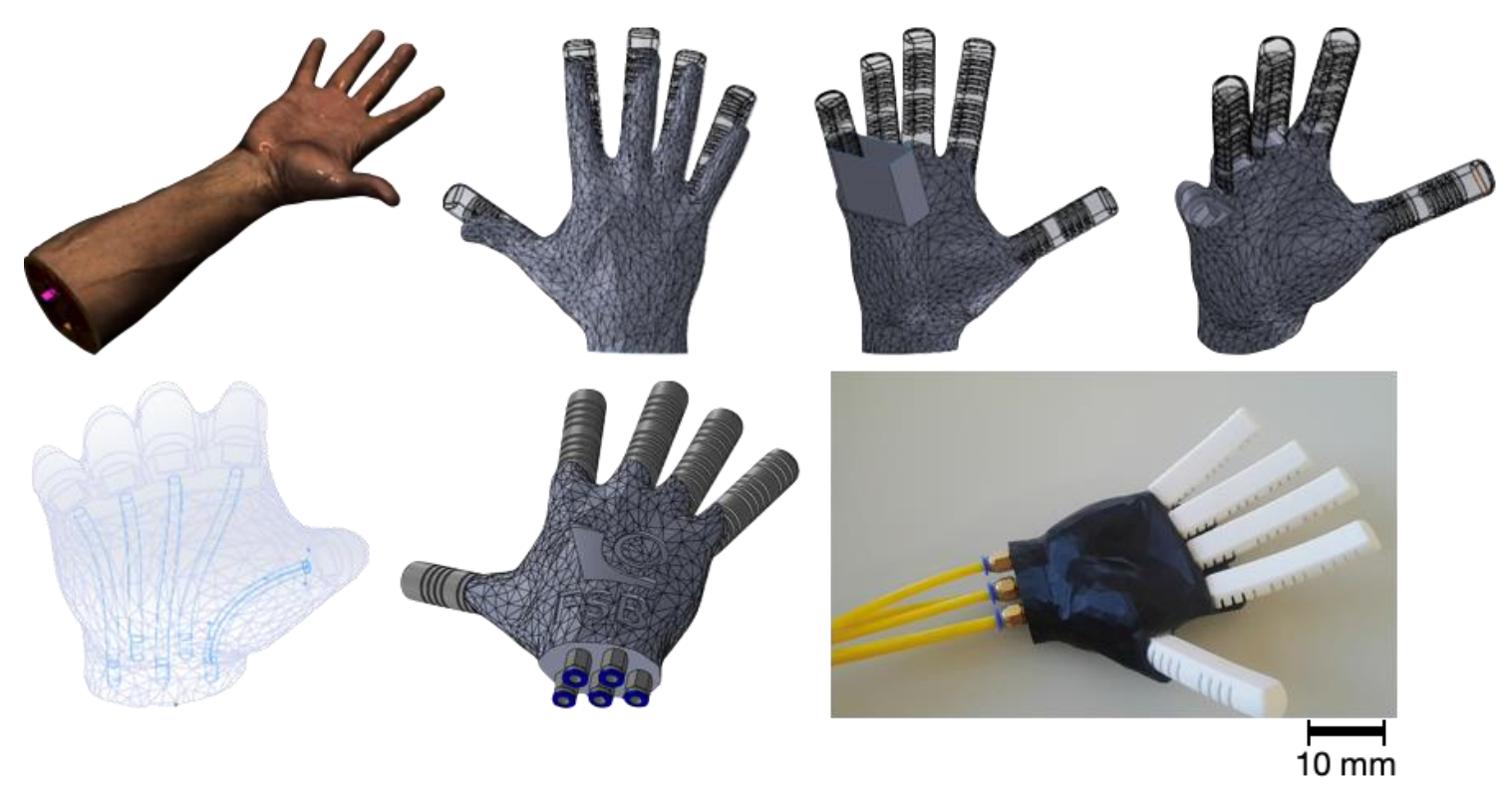

Figure 1: Anthropomorphic hand pre-processing and design: from initial model of a human hand to a finally printed and functional hand used for experimental verification.

Several simplifications of the hand presented in this study in respect to a human hand have been made. Motions in joints below carpometacarpal line are not included in analysis. Regarding the model of a human hand: it consists of 27 bones divided in following groups: 
Carpals, Metacarpals, Proximal phalanges, Intermediate phalanges, and Distal phalanges. Of these, the last three belong to the fingers, with the distinction of the thumb, which does not have the intermediate phalange [27]. Between the bones, the joints are located, and named according to the bones that they connect: Carpometacarpal (CMC); Metacarpophalangeal joints (MCP); Proximal Interphalangeal joints (PIP), and Distal interphalangeal joints (DIP) thumb has also Interphalangeal joint (IP). When analysing models of human hand in terms of technical artifacts, usually several joints are neglected so that the total number is less than twenty [28].

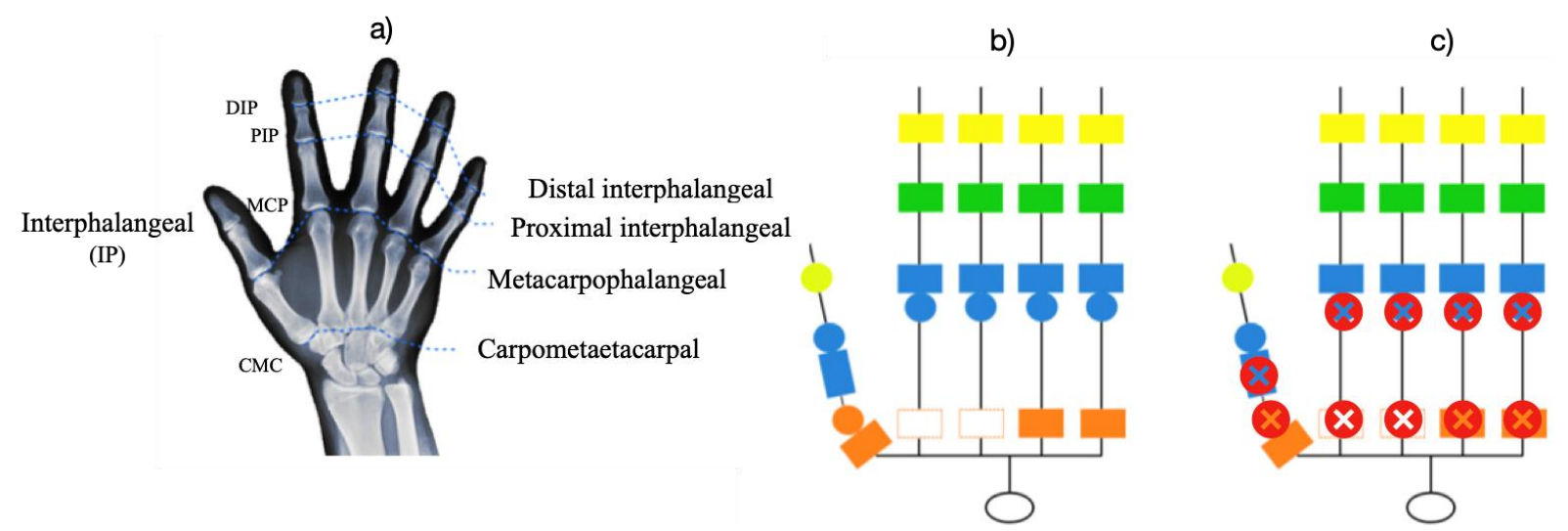

Figure 2: a) joints in human hand, b) the kinematic model, c) the simplified model.

To simplify the kinematic model of the hand, as illustrated in Fig. 2, we apply the following two rules: (I) 2 DOF joints are reduced to a single degree of freedom (CMC joint of the thumb, and all the MCP joints) with following in mind - we remove the joint less important for the opposition movement which is of the paramount importance for object manipulation. (II) due to small range of motion, all the CMC joints are neglected. Using these two rules, the total number of degrees of freedom is reduced from 25 to 15 . It is important to stress that in the case of soft robotic hand, not all 15 DOFs will be actuated, the function will be the consequence of the design of the soft actuator, i.e., of preprograming of the function into the material.

Table I: Guide for joints range of motion using defined rules.

\begin{tabular}{|l|c|c|c|c|c|}
\hline \multicolumn{1}{|c|}{ Digit } & CMC & MCP & PIP & IP & DIP \\
\hline Thumb & $50^{\circ}$ & $60^{\circ}$ & - & $80^{\circ}$ & - \\
Index & - & $80^{\circ}$ & $105^{\circ}$ & - & $70^{\circ}$ \\
Middle & - & $85^{\circ}$ & $105^{\circ}$ & - & $70^{\circ}$ \\
Ring & - & $85^{\circ}$ & $105^{\circ}$ & - & $65^{\circ}$ \\
Little & - & $85^{\circ}$ & $105^{\circ}$ & - & $70^{\circ}$ \\
\hline
\end{tabular}

Applying the rules given above, we want to design a hand to mimic the morphological motion and function of the human hand. Regarding the range of motion of fingers, we will use data given in [29], as a guide. These values vary significantly because of the discrepancies caused by individual's anatomy distribution. We give the averaged and rounded values adopted in this study in Table I.

\subsection{Theoretical analysis and simulation of proposed soft actuators}

The objective set on hand design is to allow the fingers to resemble the movement and poses of human fingers. A set of conceptual designs were created, and a simplified linear model used for initial calculations. These results are in a later phase used to define initial geometrical distribution of air chambers to enable realistic motion and yielding human-like finger poses. Parameters of interest were the layout of air chambers and the geometry of each surface of the 
finger, whereas to resemble to pose of human finger, the distribution of air chambers is particularly important.

Upon applying the pressure on the internal surface of the finger, and due to difference in material properties of various parts of the finger the part of the finger that has a higher compliance deforms excessively causing the finger to deform in approximately a circular pattern.

To achieve required rotation according to specified values, we perform analytical description of the mechanics of one finger, as illustrated in Fig. 3.
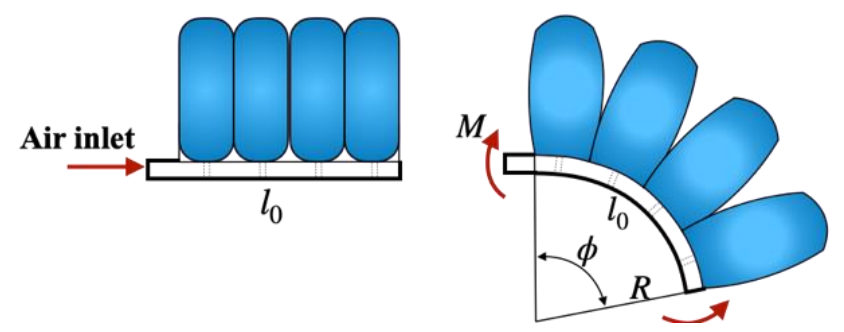

Figure 3: Analysis of the actuator for the linear model.

The total bending moment transmitted through the cross section of the actuator equals:

$$
M=\int_{A} \frac{E \cdot y^{2}}{R} d A=\frac{E}{R} \int_{A} y^{2} d A
$$

where $M$ is for bending moment, $E$ is elastic modulus, $R$ is radius of curvature, and $y$ is the distance from neutral surface. Since the integral term in the above equations on the right side defines moment of inertia $I$, the relation between bending moment and radius of curvature $R$ becomes:

$$
M=\frac{E \cdot I}{R}
$$

On the other hand, external bending moment is defined as follows:

$$
M_{p}=n \cdot p \cdot A \cdot h_{e}
$$

where $M_{p}$ is for external bending moment caused by external pressure, $n$ is the number of chambers, $p$ is for pressure, $A$ is for surface of the chamber, and $h_{e}$ is the position of the neutral axis, the location of the pressure centre.

In the case of equilibrium, and assuming the bottom part of the actuator, defined with initial length $l_{0}$ is deforming significantly less than the top part $\varepsilon_{b} \ll \varepsilon_{t}$, assuming $l_{0}=R \cdot \phi$ following expression can be formulated:

$$
\phi(n, p)=\frac{n \cdot p \cdot A \cdot h_{e} \cdot l_{0}}{E \cdot I}
$$

where $n$ is number of chambers, $p$ is pressure, $A$ is chamber surface, $h_{e}$ is the position of the neutral axis or pressure centre, $l_{0}$ is initial length of bottom part of a single chamber, $E$ is elastic modulus, and $I$ is moment of inertia.

If we want to make a full circle, by knowing the geometry of the finger, we can calculate the required number of chambers for given pressure using the expression given in Eq. (4).

Initial calculations, based on CAD (Computer Aided Design) geometry, have revealed that 7 air chambers on top layer of the finger are enough to rotate the finger for $360^{\circ}$ with internal pressure of $\sim 0.2 \mathrm{MPa}$. This pressure is selected to be in accordance with activating pressure used in other studies. This means that each air chamber adds $\sim 51^{\circ}$ rotation angle. Bearing this in mind, the approach we take to design the fingers using the required angles from the Table I is as follows: MCP joints require at least two air chambers, PIP joints require three air chambers, and DIP joints will be approximated with a single air chamber. 
It is obvious that with geometry of fingers proposed, this will enable the fingers to achieve the desired range of motion, and with increased pressure, additional forces and more reliable grasping is expected.

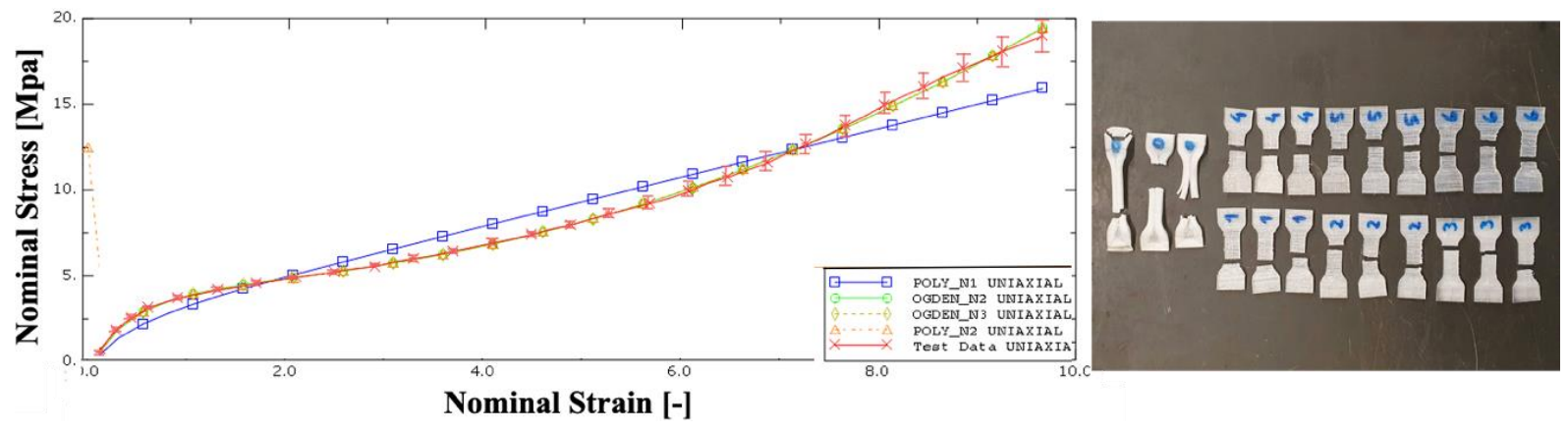

Figure 4: Comparison of different hyperelastic models as implemented in Abaqus with experimental data with confidence interval of $95 \%$ based on tensile test of specimens (right).

To validate these initial results, a simulation is performed in Abaqus for the proposed geometry. To realistically formulate the material model, we performed tensile strength test on a set of 3D printed specimens. Those data were fitted on different material models, and finally Ogden second order model was chosen as the best fit for the data, as illustrated in Fig. 4. The data from our experiments, including four different printing patterns for fused material deposition were comparable to those from [22].
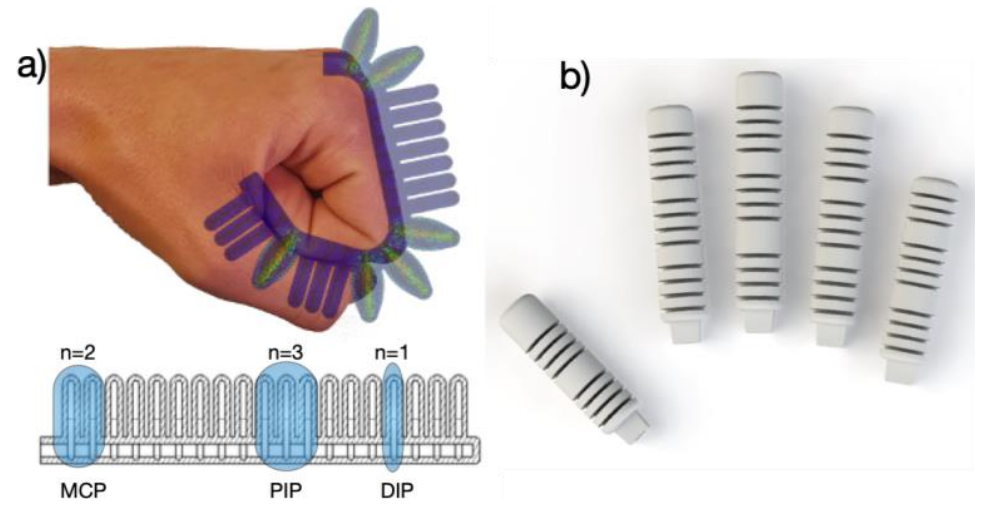

Figure 5: a) initial design of index finger, b) finally adopted design of fingers.

The conceptual design of a finger, based on the established relation between joint angle rotations and air chamber distributions, in Fig. 5 a. The simulation is performed in Abaqus using described material model, and hybrid tetrahedral C3D10H elements. MCP joint is replaced with two chambers, PIP joint with three chambers, and DIP joint with a single pressure chamber. Also, it is important to note that for the proposed design, the pressure applied was $0.15 \mathrm{MPa}$, and maximal stress was well below the yield strength of material given by manufacturer.

Despite initial results were promising, this design of a finger was not adopted as final due to used printers' limitations. Problems with stringing, warping, and porosity were pronounced so the final design was modified, Fig. 5 b. In the latter design, the pose of soft finger will not as accurately resemble the one from biological hand, which is a trade-off we had to accept due to used printers' limitations.

\subsection{Printing process limitations}

The initial design was not reliably printable using a Prusa i3 printer, as illustrated in Fig. 6 a. Thus, additional analysis was performed which enabled an increased reliability in printing of 
actuators. The approach to modification was twofold, including: (I) redesign of the fingers, and (II) tuning the process parameters of the printer. To address the former, we increased the wall thickness, and increased the fillets radii. Also, straight wall chambers were replaced with a circular wall which proved to reduce the warping of walls of air chambers. The walls were prone to collapsing in the initial design. To address the latter, parameters such as: retraction length, nozzle diameter, temperature, and fan speed were experimentally tuned.

Following conclusions have been made: retraction length set to $2 \mathrm{~mm}$, retraction speed set to $120 \mathrm{~mm} / \mathrm{s}$, fan speed: $90 \%$, layer thickness: $0.2 \mathrm{~mm}$, temperature $240{ }^{\circ} \mathrm{C}$. Nozzle diameter $0.4 \mathrm{~mm}$. Specimen on the left in Fig. $6 \mathrm{~b}$ is printed with $0.8 \mathrm{~mm}$ nozzle, specimen in the middle with $0.4 \mathrm{~mm}$ nozzle, and specimen on the right side with $0.25 \mathrm{~mm}$ nozzle. The effect of the nozzle diameter is that higher diameter causes higher rigidity, more stringing, low outer surface quality, but the volume is least porous out of all. Nozzle diameter $0.25 \mathrm{~mm}$ yields best outer surface quality, takes more time to print, induces frequent problems with filament jamming, but most importantly the volume is highly porous and thus not applicable for soft robotic applications.

a)

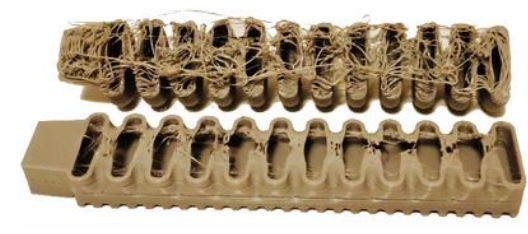

c)

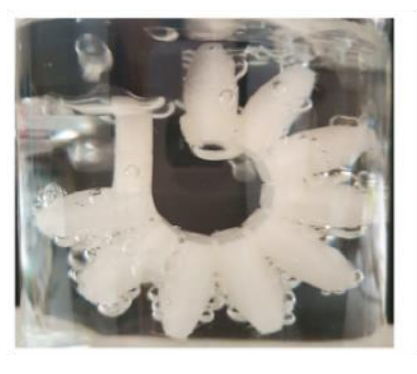

b)

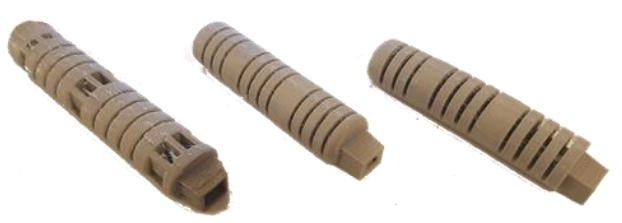

d)

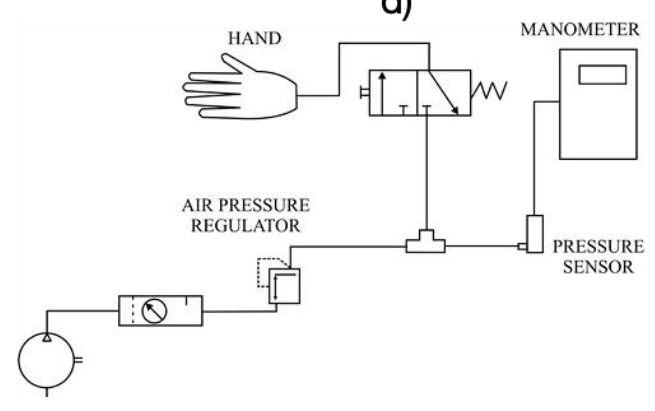

Figure 6: a) initial design, b) specimens printed with parameters tuned and design modifications, c) structural porosity, d) experimental setup.

The $0.4 \mathrm{~mm}$ nozzle was finally adopted since it produces good surface quality, structure is highly compliant, and the volume is moderately porous, as illustrated in Fig. $6 \mathrm{c}$. The porosity of material revealed to be the most significantly limiting factor in application of FDM to proposed design of soft actuator.

It was determined that air leaking increases with increase of the pressure, but the leaking is also very pronounced when curvature of the structure is large. The curvature was manipulated also manually for constant pressure to verify that porosity increases due to increased curvature. The initial experimental setup for verification of the hand is schematically illustrated in Fig. 6 d.

\section{EXPERIMENTAL VERIFICATION AND DISCUSSION}

To validate the results of the linear model and the results of the proposed hyperelastic Finite Element Method (FEM) model, the physical hand was connected to a pressure supply, and a series of experiments were performed manipulating activation pressure level.

The measurements of experimental fingertip rotation angle were performed on the images presented in Fig. 7. Currently, these images were analysed using a simple image processing routine, but advanced methods based on photogrammetry have been recently proposed to enable 
3D scanning of a human body [30]. Such methods can be used to analyse pose of all fingers simultaneously, but also for real time model reconstruction in cyber-physical domain. Both the fingertip rotation angle and the curvature of the fingers were measured. The fingers were actuated independently. The behaviour of index finger is presented.

The differences between the analytical model, and experimental data are in the range $\sim 2^{\circ}$ for initial, small pressure levels, and up to $\sim 150^{\circ}$ at pressure of $0.25 \mathrm{MPa}$. The difference increases with increase of internal pressure and increase of curvature. This is expected since nonlinearities are more pronounced in the domain of higher pressure.
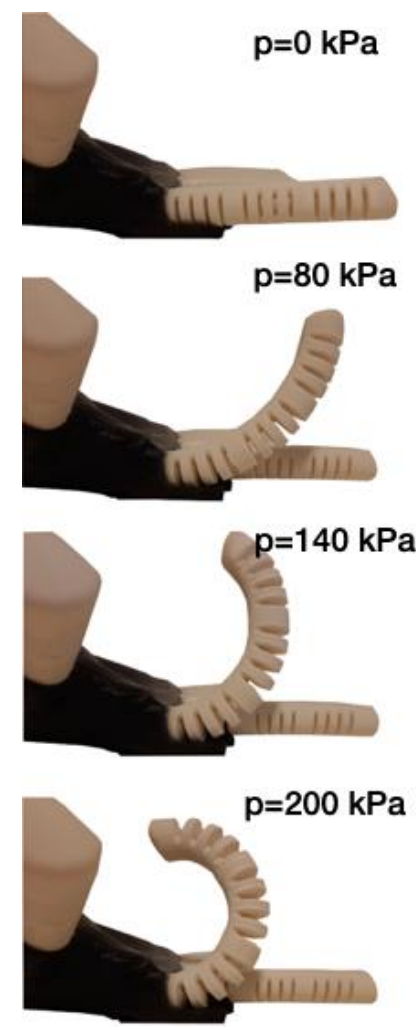
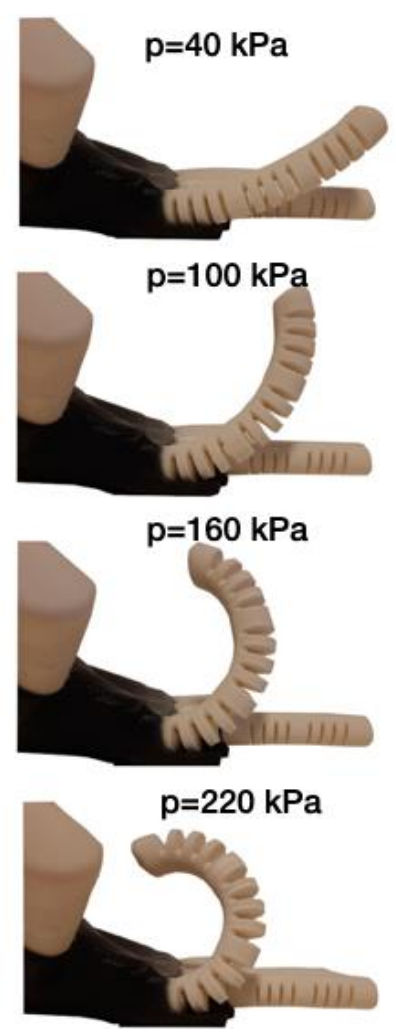
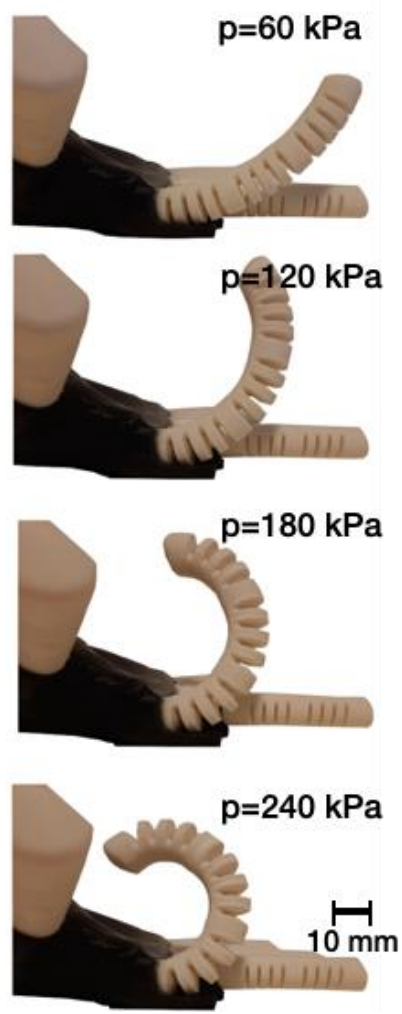

Figure 7: Experimental verification of the proposed soft anthropomorphic hand.

If we compare this data to the Ogden second order model used for simulations, it is obvious that increased pressure increases also internal stress, and that additional energy is required to achieve a constant increment of deformation. Here the maximal difference between the model and experimental data is in the range of up to $\sim 90^{\circ}$ for $0.25 \mathrm{MPa}$ pressure.

The model proposed and implemented in Abaqus, which does not include porosity of material, although significantly better than linear model, still lacks on accuracy. Additional research and fine tuning of parameters is required to achieve a better agreement between the model and actual physical actuator.

The three models are compared in Fig. 8. Several points are important here: if all the values in Eq. (4) regarding material and geometry are constant, then the relation of rotation angle to internal pressure is a linear function whose slope is defined by elastic constants and finger geometry. This is a model simplification which does not capture highly non-linear behaviour of the material. It does not capture porosity of material for higher curvatures either. Despite significant differences between the analytical model, it might be useful for initial calculations or for the scenarios where speed of calculation plays a critical role, and when operating pressure is small.

The experiments performed in this study lead to the conclusion that the main origin for the deviation of FEM model from the physical model is the leaking of pressurized air that occurs 
through the volume of the soft actuator. Experiments with submerging the finger under the water revealed that this happens along the whole surface, which is stretched, and that it is not located in regions of largest deformations or highest stresses. This also leads to a plateau in the pressure to bending ratio, which is illustrated in Fig. 8. This limits the ability of printed actuator to reach a full circle bending due to the leaking caused by increased porosity with increased curvature. Also, gravitational effects were not considered in this study, but certainly adds to the difference between the model and actual data.

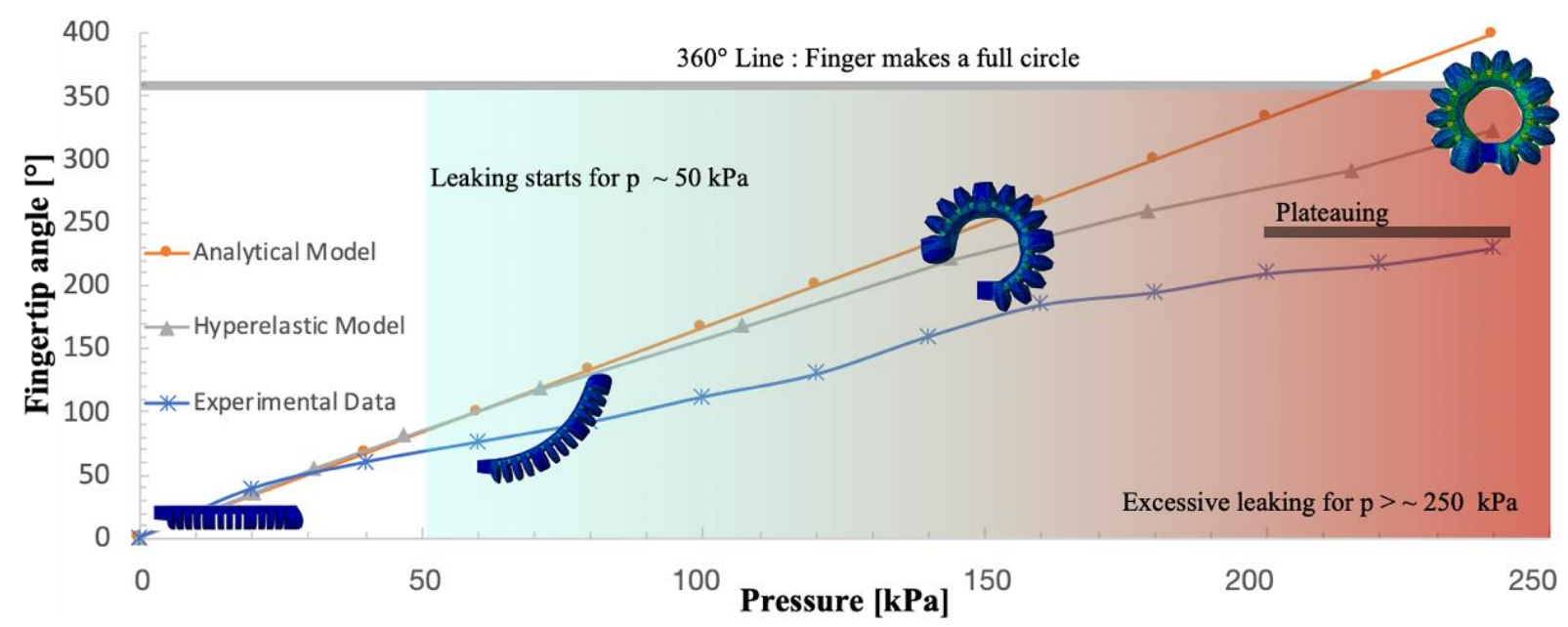

Figure 8: Comparison of experimental and analytical data for fingertip rotation. Gradient filed is to indicate leaking of air through walls of the actuator.

The proposed design was experimentally tested for grasping and lifting a variety of objects, a subset of which is illustrated in Fig. 9. The hand can reliably grasp from a flat surface objects such as apple, tomato, plastic bottle, banana, croissant, car toy model, among others without damaging the objects. The weight of manipulated objects was up to $0.3 \mathrm{~kg}$. Once reliable grasp is established, lifting and manipulating of objects is smooth.
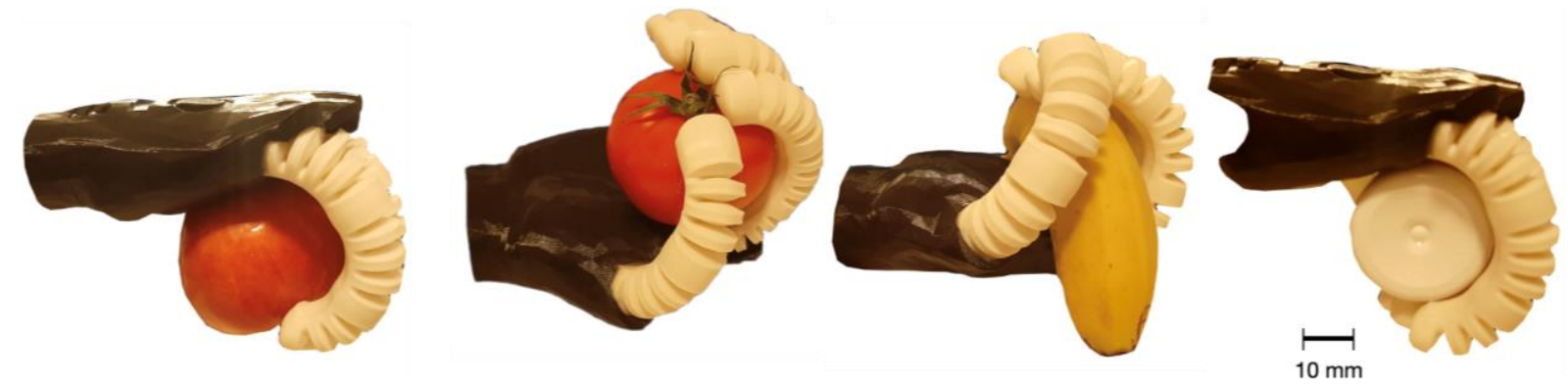

Figure 9: Grasping and manipulation of objects using proposed hand.

Objects problematic for grasping are those with one dimension significantly smaller than the two others - such as a mobile phone. For a reliable manipulation of such objects, more refined grasp should be applied in combination with additional movement and positioning, which is difficult to achieve with proposed design. Joints critical for this grasp are eliminated from the structure in the design phase - such as interphalangeal joint of the thumb. One solution to this problem would be to increase friction on fingertips. The other approach would be to include additional joints which enable fine flexion, adduction, and abduction. It is worth noting though, that the objects, once in the hand, including a mobile phone, can be reliably held, and manipulated by it. The manipulation of small objects, smaller than $1 \mathrm{~cm}^{3}$ is also not reliable. A finer sensory-motor relationship should be implemented to reliably manipulate object of such sizes. 
The next limitation of the design proposed in this study is the design of the palm manifold, which is rigid, but also has an exceptionally low friction coefficient. This generates problems in establishing the grasp, especially in the moment when the fingers are pushing the object towards the palm, have still not conformed to the objects' geometry which happens prior to lifting. If the object to be lifted is small and rigid, then sliding of the object along the palm can occur due to forces induced being not perpendicular and not oriented to the centre of gravity of the palm. These forces then push the object along the surface of the palm and the grasp is not established.

The fingers were bonded to the palm using a Loctite EA 3423 which is a 2-component structural bonding epoxy adhesive with a fixture time of $180 \mathrm{~min}$. The viscosity of this epoxy is low, $300 \mathrm{mPas}$ at $20^{\circ} \mathrm{C}$. This enables formation of a reliable bond between the small contact surfaces of the palm manifold and fingers which are subjected to internal pressure.

\section{CONCLUSIONS}

In this study, a fully operational anthropomorphic hand is designed and simulated with a linear and hyperelastic model, and finally printed using FDM low-cost open source Prusa i3 printer.

Printed specimens were tested on a universal testing machine to formulate a realistic hyperelastic material model. This model is more accurate, but significantly more time consuming compared to the linear model. Printing parameters and orientations were varied for printed specimens, which had a significant impact on their properties. Ogden second order model was determined to be the best fit for experimental data and was adopted as the model used thereafter in numerical simulations.

The soft actuators were printed using a Prusa i3 open source FDM printer, and material used was NinjaFlex TPU. Printed actuators were bonded to a rigid manifold to resemble the anthropomorphic hand consisting of fingers, thumb, and a palm. This enabled the experimental verification of the data from simulation models. The hand was used both to lift and manipulate a set of objects. Measuring of fingertip rotation angle along with curvature depending on activating pressure was performed.

The difference in fingertip rotation angle between the physical finger and linear model is $150^{\circ}$ max. The difference in fingertip rotation angle between physical finger and proposed FEM model is in the range of up to $\sim 90^{\circ}$ max. In the latter case, the soft actuator in the simulation environment almost closes a full circle, whereas experimental finger closes $\sim 260^{\circ}$. Despite the difference is large, it occurs on the higher end of the pressure range, with large curvatures of the actuator achieved for both bending angles. In the rest of the working range, the difference is significantly smaller.

The properties of 3D printed hand in this study were constrained by the nature of FDM approach itself, but also by the printer used for the fabrication of the fingers. Initially proposed designs were not reliably printable, and some features of initial fingers were omitted in the final design. A thorough experimental validation and comparison with both numerical and FEM analysis were performed.

FEM model formulated in this study can capture non-linearities of the actual physical model. The differences between real model and FEM calculations can have roots both in not perfectly determined parameters for the hyperelastic Ogden model, but also a significant impact on accuracy is because of plateauing which occurs for large curvatures and increased porosity of material. The voids which are present in the volume of soft actuator manufactured by FDM process used here are not implemented and will be a focus in a future research.

Another important aspect for future research is the evaluation of properties of different materials used for palm manifold, but also for the fingers. The friction coefficient should be high to establish reliable grasp without sliding. The geometry of fingers should also be modified 
to exhibit more concentric movement of the fingers toward the centre of mass of the palm manifold, instead of circular movement towards carpometacarpal region which occurs with present design. This would help in reducing the sliding of objects in contact with palm and being pushed by the fingers simultaneously.

Finally, there is a need for regulation of soft fingers angle of rotation with respect to external pressure applied. This would enable a wider range of applications, reduce energy used for grasping, and enable even more organic motion of the fingers.

\section{ACKNOWLEDGEMENT}

This study was partially supported by a Fulbright Visiting Scholar Grant No. 68140072 for P. Ć. The author kindly acknowledges the hospitality of Professor Hod Lipson in the Creative Machines Lab.

\section{REFERENCES}

[1] Polygerinos, P.; Wang, Z.; Overvelde, J. T. B.; Galloway, K. C.; Wood, R. J.; Bertoldi, K.; Walsh, C. J. (2015). Modeling of soft fiber-reinforced bending actuators, IEEE Transactions on Robotics, Vol. 31, No. 3, 778-789, doi:10.1109/TRO.2015.2428504

[2] Joshi, S.; Paik, J. (2020). Pneumatic supply system parameter optimization for soft actuators, Soft Robotics, Vol. 8, No. 2, 152-163, doi:10.1089/soro.2019.0134

[3] Curkovic, P.; Jambrecic, A. (2020). Improving structural design of soft actuators using finite element method analysis, Interdisciplinary Description of Complex Systems, Vol. 18, No. 4, 490500, doi:10.7906/indecs.18.4.8

[4] Polygernios, P.; Cornell, N.; Morin, S. A.; Mosadegh, B.; Cagdas, D. O.; Petersen, K.; Cianchetti, M.; Tolley, M. T.; Shepherd, R. F. (2017). Soft robotics: review of fluid-driven intrinsically soft devices: manufacturing, sensing, control, and applications in human-robot interaction, Advanced Engineering Materials, Vol. 19, No. 12, Paper 1700016, 22 pages, doi:10.1002/adem.201700016

[5] Chellttoan, R.; Yudhanto, A.; Lubineau, G. (2020). Low-voltage-driven large-amplitude soft actuators based on phase transition, Soft Robotics, Vol. 7, No. 6, 688-699, doi:10.1089/ soro.2019.0150

[6] Brown, E.; Rodenberg, N.; Amend, J.; Mozeika, A.; Steltz, E.; Zakin, M. R.; Lipson, H.; Jaeger, H. M. (2010). Universal robotic gripper based on the jamming of granular material, PNAS, Vol. 107, No. 44, 18809-18814, doi:10.1073/pnas.1003250107

[7] Marchese, A. D.; Onal, C. D.; Rus, D. (2014). Autonomous soft robotic fish capable of escape manoeuvres using fluidic elastomer actuators, Soft Robotics, Vol. 1, No. 1, 75-87, doi:10.1089/soro.2013.0009

[8] Ge, G.; Huang, W.; Shao, J.; Dong, X. (2018). Recent progress of flexible and wearable strain sensors for human motion monitoring, Journal of Semiconductors, Vol. 39, No. 1, Paper 011012 , 21 pages, doi: $10.1088 / 1674-4926 / 39 / 1 / 011012$

[9] Scharff, R. B. N.; Wu, J.; Geraedts, J. M. P.; Wang, C. C. L. (2019). Reducing out-of-plane deformation of soft robotic actuators for stable grasping, Proceedings of the $2^{\text {nd }}$ IEEE International Conference on Soft Robotics, 265-270, doi:10.1109/ROBOSOFT.2019.8722823

[10] Park, T.; Cha, Y. (2019). Soft mobile robot inspired by animal-like running motion, Scientific Reports, Vol. 9, Paper 14700, 9 pages, doi:10.1038/s41598-019-51308-4

[11] Curkovic, P. (2021). Optimization of generatively encoded multi-material lattice structures for desired deformation behavior, Symmetry, Vol. 13, No. 2, Paper 293, 13 pages, doi: $10.3390 /$ sym 13020293

[12] Chu, C.-Y.; Patterson, R. M. (2018). Soft robotic devices for hand rehabilitation and assistance: a narrative review, Journal of Neuro Engineering and Rehabilitation, Vol. 15, Paper 9, 14 pages, doi:10.1186/s12984-018-0350-6

[13] Yap, H. K.; Lim, J. H.; Goh, J. C. H.; Yeow, C.-H. (2016). Design of a soft robotic glove for hand rehabilitation of stroke patients with clenched fist deformity using inflatable plastic actuators, Journal of Medical Devices, Vol. 10, No. 4, Paper 044504, 6 pages, doi:10.1115/1.4033035 
[14] Runciman, M.; Darzi, A.; Mylonas, G. P. (2019). Soft robotics in minimally invasive surgery, Soft Robotics, Vol. 6, No. 4, 423-443, doi:10.1089/soro.2018.0136

[15] Sanchez, V.; Walsh, C. J.; Wood, R. J. (2021). Textile technology for soft robotics and autonomous garments, Advanced Functional Materials, Vol. 31, No. 6, Paper 2008278, 55 pages, doi:10.1002/adfm.202008278

[16] Zhou, Y. M.; Wagner, D.; Nuckols, K.; Heimgartner, R.; Correia, C.; Clarke, M.; Orzel, D.; O’Neill, C.; Solinsky, R.; Paganoni, S.; Walsh, C. J. (2019). Soft robotic glove with integrated sensing for intuitive grasping assistance post spinal cord injury, Proceedings of the 2019 International Conference on Robotics and Automation, 9059-9065, doi:10.1109/ICRA.2019.8794367

[17] Jin, Y.; Glover, C. M.; Cho, H.; Aorami, O. A.; Graule, M. A.; Li, N.; Wood, C. J.; Walsh, C. J. (2020). Soft sensing shirt for shoulder kinematics estimation, Proceedings of the 2020 IEEE International Conference on Robotics and Automation, 4863-4869, doi:10.1109/ ICRA40945.2020.9196586

[18] Vasios, N.; Gross, A. J.; Soifer, S.; Overvelde, J. T. B.; Bertoldi, K. (2020). Harnessing viscous flow to simplify the actuation of fluidic soft robots, Soft Robotics, Vol. 7, No. 1, 1-9, doi:10.1089/soro.2018.0149

[19] Moseley, P.; Florez, J. M.; Sonar, H. A.; Agarwal, G.; Curtin, W.; Paik, J. (2015). Modeling, design, and development of soft pneumatic actuators with finite element method, Advanced Engineering Materials, Vol. 18, No. 6, 978-988, doi:10.1002/adem.201500503

[20] Yap, Y. L.; Sing, S. L.; Yeong, W. Y. (2020). A review of 3D printing processes and materials for soft robotics, Rapid Prototyping Journal, Vol. 26, No. 8, 1345-1361, doi:10.1108/RPJ-11-2019$\underline{0302}$

[21] Yap, H. K.; Ng, H. Y.; Yeow, C.-H. (2016). High-force soft printable pneumatics for soft robotic applications, Soft Robotics, Vol. 3, No. 3, 144-158, doi:10.1089/soro.2016.0030

[22] Reppel, T.; Weinberg, K. (2018). Experimental determination of elastic and rupture properties of printed NinjaFlex, Technische Mechanik, Vol. 38, No. 1, 104-112, doi:10.24352/UB.OVGU-2018010

[23] NinjaTek Technical Specifications. NinjaFlex 3D Printing Filament, from https://ninjatek.com/wpcontent/uploads/2019/10/NinjaFlex-TDS.pdf, accessed on 19-01-2021

[24] Tronvoll, S. A.; Welo, T.; Elverum, C. W. (2018). The effects of voids on structural properties of fused deposition modelled parts: a probabilistic approach, The International Journal of Advanced Manufacturing Technology, Vol. 97, No. 9-12, 3607-3618, doi:10.1007/s00170-018-2148-X

[25] Tronvoll, S. A.; Vedvik, N. P.; Elverum, C. W.; Welo, T. (2019). A new method for assessing anisotropy in fused deposition modelled parts using computed tomography data, The International Journal of Advanced Manufacturing Technology, Vol. 105, No. 1-4, 47-65, doi:10.1007/s00170019-04081-7

[26] Lopes, H.; Silva, S. P.; Machado, J. (2020). Simulation of temperature evolution of cork composites during moulding process, International Journal of Simulation Modelling, Vol. 19, No. 4, 583-594, doi:10.2507/IJSIMM19-4-530

[27] Schmidt, H.-M.; Lanz, U. (2004). Surgical anatomy of the hand, Journal of Hand Surgery (European Volume), Vol. 29, No. 4, 410-411, doi:10.1016/J.JHSB.2004.04.001

[28] Piazza, C.; Grioli, G.; Catalano, M.; Bicchi, A. (2019). A century of robotic hands, Annual Review of Control, Robotics, and Autonomous Systems, Vol. 2, No. 1, 1-32, doi:10.1146/annurev-control060117-105003

[29] Lee, K.-S.; Jung, M.-C. (2015). Ergonomic evaluation of biomechanical hand function, Safety and Health at Work, Vol. 6, No. 1, 9-17, doi:10.1016/j.shaw.2014.09.002

[30] Gajic, D. B.; Mihic, S.; Dragan, D.; Petrovic, V.; Anisic, Z. (2019). Simulation of photogrammetrybased 3D data acquisition, International Journal of Simulation Modelling, Vol. 18, No. 1, 57-71, doi: $\underline{10.2507 / \operatorname{IJSIMM} 18(1) 460}$ 